\title{
Philosophiques
}

\section{Réponse à Yvon Gauthier, Louis Marchildon et Serge Robert \\ Storrs McCaII, A Model of the Universe, New York, Oxford University Press, 1994}

\section{Storrs McCaIl}

Volume 22, numéro 2, automne 1995

URI : https://id.erudit.org/iderudit/027350ar

DOI : https://doi.org/10.7202/027350ar

Aller au sommaire du numéro

Éditeur(s)

Société de philosophie du Québec

ISSN

0316-2923 (imprimé)

1492-1391 (numérique)

Découvrir la revue

Citer cet article

McCaIl, S. (1995). Réponse à Yvon Gauthier, Louis Marchildon et Serge Robert /

Storrs McCaII, A Model of the Universe, New York, Oxford University Press,

1994. Philosophiques, 22(2), 489-503. https://doi.org/10.7202/027350ar d'utilisation que vous pouvez consulter en ligne.

https://apropos.erudit.org/fr/usagers/politique-dutilisation/ 


\title{
RÉPONSE À YVON GAUTHIIER, LOUIS MARCHIDON ET SERGE ROBERT
}

\author{
par Storrs McCall
}

\section{A. Yvon Gauthier et Louis Marchildon}

Ces deux chercheurs ont fait un travail admirable et ont écrit des commentaires pertinents et pénétrants de mon livre, $A$ Model of the Universe. Dans plusieurs cas, leurs observations concernent le même sujet, et je vais donc les traiter ensemble.

Dans son excellente revue, Louis Marchildon affirme que le modèle est contradictoire dans deux de ses aspects. Il dit aussi que les explications fournies par le modèle sont le plus souvent illusoires. Ces conclusions reflètent un jugement sévère. Certes, si j'avais pensé à l'époque où j'écrivais l'ouvrage que le modèle qui y joue le rôle principal donnait lieu à des contradictions, ou foumissait des explications illusoires, j'aurais immédiatement jeté mon manuscrit à la poubelle. Un modèle qui prétend être exact et rigoureux mais qui contient des contradictions ne vaut rien. Pour cette raison, nous devons examiner le modèle avec la plus scrupuleuse attention pour voir si, en effet, les contradictions entrevues par Marchildon existent ou non.

\section{Les contradictions dans le modèle}

À la page 2 de son commentaire, Marchildon cherche à comprendre comment la structure quadridimensionnelle de l'univers à son début peut différer de sa structure quadridimensionnelle ultérieure. Il dit, en effet, que ceci est impossible puisque :

1. «Affirmer que l'univers quadridimensionnel a telle ou telle structure, c'est affirmer de facto la structure de l'espace tridimensionnnel (ou des espaces tridimensionnels s'il y en a plusieurs) à tout instant "

et

2. «Il est contradictoire de dire que l'espace à tout instant, à $t_{2}$, est différent de l'espace à tout instant, à $t_{1}$ ".

Si les propositions 1 et 2 étaient vraies, il serait contradictoire de conclure que la structure quadridimensionnelle de l'univers à un temps postérieur est une sous-structure de la structure correspondant à un temps antérieur, la différence résultant de l'émondage des branches. 
Mais les propositions 1 et 2 ne sont pas vraies. Pour voir la raison pour laquelle il en est ainsi, considérons les deux structures suivantes :
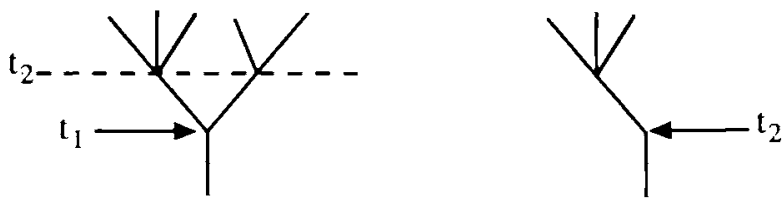

Rappelons que, dans le modele dont nous parlons, il y a un émondage progressif des branches et que l'état présent du monde se trouve toujours au premier noeud de branchement. La date de ce noeud est donnée par sa coordonnée de temps. Donc, la date du présent dans la première structure est $t_{l}$, et dans la deuxième la date du présent est $t_{2}$. C'est évident que l'espace à tout instant dans la deuxième structure, à $t_{2}$, est bien différent de l'espace à tout instant dans la première structure, à $t_{l}$. Par exemple, dans la première structure, il y a deux espaces tridimensionnels différents à $t_{2}$ tandis que, dans la deuxième structure, l'espace tridimensionnel à $t_{2}$ est unique. Le modèle arborescent est un modèle dynamique, non statique, et sa structure quadridimensionnelle change d'un moment à l'autre. Par conséquent, la structure de l'espace tridimensionnel à tout instant varie aussi.

Passons maintenant à la deuxième contradiction identifiée par Marchildon dans le modèle. Celle-ci regarde la manière dont le branchement s'opère dans le modèle, en particulier la question de savoir si le processus de branchement réintroduit « la simultanéité absolue que la relativité restreinte avait éliminée » (p. 7). Gauthier aussi a fait des observations sur ce point. Marchildon dit, en effet, que si le branchement s'effectue suivant l'hypersurface où $t$ est une constante dans un référentiel donné, il ne peut pas aussi s'effectuer suivant une autre hypersurface où $t^{\prime}$ est une constante dans un autre référentiel. Il affirme, ce qui est parfaitement vrai, qu'il n'existe pas de transformation de Lorentz qui peut transformer une structure qui branche suivant la première surface en une structure qui branche suivant la deuxième surface. Pour le dire dans ses mots, une transformation de Lorentz " ne fait pas disparaître d'objets ». La transformation en question étant non existante, il conclut que le processus du branchement qui s'opère dans le modèle introduit un référentiel privilégié, contrairement à l'esprit de la théorie de la relativité.

Avec ce problème d'un référentiel privilégié, Marchildon soulève la question à laquelle $\mathrm{j}$ 'ai consacré presque toutes mes énergies depuis la publication du livre. Il est vrai qu'il faut éviter d'adopter un référentiel privilégié. Mais justement, le modele n'est pas basé sur un tel référentiel. Au contraire, le processus de branchement se poursuit selon tous les référentiels à la fois. $\mathrm{La}$ situation est compliquée, et je ne peux donner qu'un sommaire de mes résultats. L'objectif est de démontrer qu'il existe une parfaite cohérence entre le modèle et la théorie de la relativité. 
En bref, le modèle est un énorme espace topologique composé de pointsévénements. Cette espace differe d'un espace-temps de Minkowski par le fait qu'il contient des paires de points qui sont inaccessibles l'un à l'autre, c'est-àdire des points qui sont reliés seulement par un chemin qui commence avec un point, remonte vers le passé, et puis repart sur une autre branche. Une branche, c'est-à-dire un ensemble maximal de points qui sont mutuellement accessibles, est un espace de Minkowski. Chaque branche intersecte chaque autre branche, et la surface limite de leur intersection est une hypersurface que j'appelle une surface ondulée. Correspondant à chaque référentiel, il y a des surfaces ondulées qui sont parallèles à des hypersurfaces où $t$ est une constante dans le référentiel en question. Le branchement du modèle se poursuit le long de chacune de ces surfaces ondulées.

J'appelle l'ensemble total des points-événements du modèle " $\mathbf{W}$ » ou, mieux, "Grand-W ». Naturellement, puisque les surfaces ondulées dans Grand-W se croisent, s'intersectent, et s'étendent dans toutes les directions, le mode de branchement de l'espace Grand-W est chaotique. Grand-W ne ressemble pas du tout aux structures arborescentes régulières et bien ordonnées qu'on retrouve dans les pages de $A$ Model of the Universe. Néanmoins, ces structures régulières existent comme sous-ensembles de Grand-W. Grand-W est une structure caractérisée par l'invariance de Lorentz. Mais si on choisit un référentiel $f$ dans lequel le temps du point ici-maintenant est $t$, les deux paramètres $f$ et $t$ déterminent un unique sous-ensemble $\mathbf{W} f t$ de $\mathbf{W}$ dans lequel

(i) tout branchement se poursuit le long des surfaces ondulées parallèles aux hypersurfaces où $t$ est une constante dans le référentiel $f$ et

(ii) le point ici-maintenant se trouve au premier noeud.

C'est seulement dans ces sous-ensembles $\mathbf{W} f t$, et non dans Grand-W, que l'écoulement du temps est miroité par l'émondage régulier et successif des branches. Puisqu'il s'agit d'un sous-ensemble distinct pour chaque référentiel, il n'est pas question d'un référentiel privilégié.

\section{L'illusion d'explication}

\section{(a) La flèche du temps}

L'explication fournie pour le passage du temps, c'est-à-dire l'émondage progressif des branches dans les sous-modèles $\mathbf{W} f t$, ne donne pas lieu à une contradiction. Il est vrai que la notion d'émondage n'est pas familière aux philosophes des sciences ni reconnue par eux, mais ceci ne veut pas dire qu'elle soit contradictoire. Elle est nouvelle, certes, mais cohérente en ellemême.

Quant à l'expérience du passage du temps, Marchildon a raison quand il écrit à la page 4 de son texte que, dans le modele arborescent, il n'y a aucune relation entre la structure du branchement et l'expérience psychologique du temps. Par conséquent, Marchildon affirme que ni la structure, ni l'émondage 
des branches ne peuvent servir comme explication de notre expérience du passage du temps ou de l'asymétrie du temps. Ceci est vrai. Mais pour un philosophe, il y a d'autres sortes d'explications, ou peut-être d'autres sens du mot " explication ", que l'explication de notre expérience ou de nos états psychologiques. Bien sûr, la philosophie s'intéresse à foumir des explications de nos expériences, mais elle s'intéresse aussi à expliquer en quoi consiste telle ou telle chose ou tel ou tel phénomène. Un philosophe peut demander, par exemple : « qu’est-ce que le passage du temps? », ou « en quoi consiste l’asymétrie du temps? " En posant ces questions, il ne cherche pas à comprendre pourquoi il a telle ou telle expérience, mais il veut savoir quelle est la nature même du temps; il cherche en particulier à comprendre le caractère asymétrique du temps et la propriété liée au fait qu'il s'écoule. Le philosophe cherche une explication ontologique ou métaphysique plutôt qu'une explication psychologique, et c'est justement cette sorte d'explication que le modele essaie de foumir.

De son côté, Gauthier demande : «Comment réintroduire une dynamique dans cet univers statique? " La réponse du modèle est la suivante: "par l'émondage des branches ». Et Gauthier conclut : "Métaphysique et métaphore se rejoignent ici dans un joyeux transport. "C'est ainsi que la flèche du temps est représentée dans le modèle arborescent.

\section{(b) La notion de probabilité}

En définissant la probabilité d'un événement $E$, relativement à des conditions initiales données, par la proportion des branches qui contiennent $E$, le modele donne lieu à un concept de la probabilité qui se distingue de chacune des définitions «traditionnelles » de la probabilité, c'est-à-dire les définitions logique, fréquentielle, subjective et en termes de propensités. Mais serait-il possible, comme le soutient Marchildon, que la nouvelle définition ne nous offre qu'une conception «artificielle » des probabilités? Pour y voir plus clair, prenons le cas d'un dé jeté plusieurs fois sur une surface plate. Dans le modele arborescent, il y a six sortes de branches relatives à ces conditions initiales, une pour chacun des résultats $1,2,3,4,5,6$. La proportion relative de chaque sorte est 1/6. Est-ce une pure coincidence que la fréquence relative d'un résultat donné, mettons le résultat « trois », soit égale à la proportion relative des branches contenant le même résultat ? Sûrement pas. Le lien entre proportion de $1 / 6$ et fréquence de 1/6 est expliqué par le fait qu'à chaque fois le choix de l'unique branche actuelle est aléatoire, un résultat du hasard objectif. Étant donné la nature aléatoire de ce processus de sélection, la proportionnalité explique la fréquence relative.

Marchildon paraît reconnaître que la fréquence peut être expliquée par la proportionnalité, mais il remarque que ce fait annule la possibilité de donner une nouvelle définition de la probabilité. Si le choix de la branche actuelle est aléatoire, cela veut dire que chaque branche possède une probabilité égale d'être choisie. Donc la définition échoue. Marchildon a raison : le modèle ne 
réussit pas à définir la probabilité de novo. Mais cela n'empêche pas que le modèle serve à donner une interprétation physique de la probabilité, basée sur une combinaison de (i) la proportionnalité de différentes sortes de branche, et (ii) le hasard objectif. Contrairement à ce que dit Gauthier à la page 4 de son texte, ce n'est pas une théorie subjectiviste. Mais Gauthier a par ailleurs raison de dire que c'est une théorie sans observateur.

Cette explication des valeurs des probabilités n'est ni artificielle ni illusoire. Il y a un élément factuel et empirique dans ces valeurs, qu'on observe à la suite d'une longue série d'expérimentations, et la contribution du modèle arborescent est précisément de rendre compte de la valeur exacte de laquelle s'approche la fréquence relative. Cette valeur est enchâssée dans la structure d'espace-temps du modele. Pour illustrer mon point, je propose un pari à Louis Marchildon. On observe dans la dégénérescence des particules et atomes non stables une propriété qui est remarquablement constante et prévisible, c'est-à-dire la demi-vie. Un neutron libre, par exemple, possède une demi-vie d'environ 1000 secondes, ce qui veut dire que la probabilité qu'un neutron quelconque cesse d'exister après 1000 secondes est 0,5 . Qu'est-ce qui lui donne cette probabilité ? La réponse du modèle arborescent est claire. A chaque instant, la proportion de branches sur lesquelles un neutron libre persiste dans l'existence après 1000 secondes est toujours de $50 \%$. « La probabilité » est ici expliquée par « la proportion». Si Marchildon peut donner une autre explication, par exemple déduire la durée de la demi-vie en partant d'un point de départ exclusivement théorique, il gagnera le pari et je lui payerai alors cinq dollars. Mais si la valeur de la demi-vie s'avère incalculable au moyen de la seule théorie pure et exige le recours à l'observation, c'est-à-dire comporte un élément factuel irréductible, alors Louis Marchildon devra considérer que l'explication de cette valeur précise peut seulement être fournie par la structure du modèle arborescent. Et dans ce cas, je gagnerai le pari.

\section{(c) Causalité et nécessité physique.}

Si $A$ est suivi par $B$ sur toutes les branches, partout dans le modele, on dit que $A$ entraîne $B$ nécessairement. Il ne s'agit pas ici de nécessité logique, mais de nécessité causale ou physique. Il est sans doute logiquement possible que l'événement correspondant au moment où je laisse tomber une pièce de 25 cents soit suivi par l'événement défini par le fait que la pièce se transforme en oiseau et s'envole par la fenêtre, mais ceci n'est pas physiquement possible.

Marchildon dispute l'identification de la "nécessité physique " au fait d'être «présent sur toutes les branches du modèle ». Dans le cas de l'univers de Minkowski, qui est pour lui un exemple d'un modèle à seulement une branche, on peut toujours dire que $A$ n'entraîne pas $B$ nécessairement parce que l'univers de Minkowski aurait pu être autrement. Pourquoi pas, par analogie, dire que $A$ n'entraîne pas $B$ parce que le modèle arborescent aurait pu être autrement? Cette question est intéressante et philosophiquement subtile, et j'essaierai d'y répondre. 
(i) Premièrement, l'expression « aurait pu être autrement " de Marchildon doit être prise, si je le comprends bien, dans le sens de « aurait pu logiquement être autrement ". Si cela était correct, le seul cas où $A$ entraînerait $B$ serait le cas où $A$ impliquerait $B$ logiquement, c'est-à-dire dans tous les univers possibles, et la différence entre nécessité physique et nécessité logique disparaîtrait.

(ii) Oui, l'univers arborescent aurait logiquement pu être différent, mais ceci ne nous dit rien sur la question de ce qui est physiquement possible et physiquement nécessaire. La question qui nous intéresse ici, c'est la question de savoir ce qui est physiquement ou causalement possible, par comparaison avec ce qui est causalement nécessaire, dans notre univers, l'univers dans lequel nous vivons. La réponse à cette question se trouve dans la structure du modèle arborescent, qui contient toutes les branches, mais seulement les branches, qui sont physiquement possibles dans notre univers.

\section{(d) Les propriétés essentielles}

Le modèle définit une propriété essentielle d'un individu comme une propriété que cet individu possède sur toutes les branches et à chaque instant où l'individu existe. Si, au moment où je commence à exister, aucune branche ne me montre avec six doigts à la main gauche (l'exemple est de David Lewis), c'est alors une de mes propriétés essentielles que je n'ai pas six doigts. Dans l'exemple de Marchildon, si aucun cheveu de Samson ne vire spontanément du noir au blond sur aucune branche, c'est sans doute parce que ce changement va à l'encontre d'une loi de la nature, et le fait que la couleur de ses cheveux ne change pas de cette façon est également une des propriétés essentielles de Samson. Je suis d'accord avec Marchildon que ceci n'est pas un exemple typique d'une propriété essentielle comme ce qui détermine l'appartenance d'un individu à une espèce biologique, ou encore ce qui en détermine l'origine, mais même les propriétés plus modestes des individus, comme l'absence de changement brusque dans la couleur de leurs cheveux, méritent de n'être pass négligées.

\section{Les probabilités irrationnelles}

Marchildon donne une description claire et précise de la façon dont les probabilités irrationnelles sont représentées dans le modèle arborescent. Dans chaque intervalle temporel $\Delta t$, le modèle donne lieu à une infinité non dénombrable de branches, parmi lesquelles il existe des proportions fixes. Marchildon demande pourquoi ces " prismes " se réalisent selon un fractionnement décénaire (c.-à-d. comme un arbre à dix branches) plutôt que binaire ou ternaire, car, bien sûr, cela n'est pas nécessaire. La nature pourrait choisir n'importe quelle sorte de prisme, à condition que le prisme conserve la proportionnalité des branches - c'est-à-dire qu'à chaque niveau, le prisme se scinde en un nombre égal de branches. Marchildon demande aussi pourquoi les sous-embranchements à l'intérieur des prismes se produisent à un instant particulier plutôt qu'à un autre. Sur ce point il a entièrement raison : pourvu 
qu'il y ait une infinité dénombrable de ces instants à l'intérieur de chaque prisme, les points de sous-embranchement pourraient être distribués n'importe où.

Au sujet de la dégénérescence radioactive, Marchildon se montre sceptique sur la question de savoir si un tel processus continu, qui est d'ailleurs stochastique, pourrait être représenté par une série discrète de prismes. Rappelons que la durée temporelle de chaque prisme est une quantité finie $\Delta t$, et que chaque particule non stable possède une probabilité fixe de dégénérer au cours de l'intervalle $\Delta t$. Malheureusement, je n'ai pas réussi à trouver un modele qui donne à une particule une probabilité constante de dégénérer à chaque instant, mais seulement dans chaque intervalle $\Delta t$. Si Marchildon pouvait me suggérer un moyen d'assigner à une particule une probabilité objective et continue, par unité de temps, plutôt que discrète, j'en serais très heureux. Mais, au moment présent, je ne sais pas comment envisager un processus objectif et stochastique qui ne procède pas par petits sauts de $\Delta t$.

\section{La mécanique quantique}

Le modele arborescent essaie de donner une interprétation physique et réaliste de la version de la mécanique quantique qui postule, avec von Neumann, deux sortes d'évolution temporelle, c'est-à-dire (i) l'évolution de Schrödinger, et (ii) la réduction du vecteur d'état occasionnée par un processus de mesure. La ressemblance avec l'interprétation dite « de Copenhague » est évidente. Gauthier vise juste quand il dit qu'on assiste, dans un processus de mesure, à un passage du possible à l'actuel. C'est comme ça, du reste, que Heisenberg voyait l'affaire.

Je regrette d'avoir abrégé dans mon livre la description du processus $R$ (la mesure) pour pouvoir l'introduire du même coup dans deux domaines différents, à savoir le domaine précis de la mécanique quantique, mais aussi celui où se pose la question philosophique de l'indéterminisme. Marchildon observe, très justement, que, quand il s'agit d'un processus de mesure en mécanique quantique, les états finaux qui se trouvent sur les branches doivent toujours être des élats orthogonaux de l'observable mesurée (ou bien des produits tensoriels de ces états avec des états de l'appareil). J'ai eu tort de ne pas avoir mentionné cela. Mais je voulais souligner la ressemblance entre la mesure en mécanique quantique et tout autre phénomène contingent, par exemple l'effondrement d'un pont secoué par un tremblement de terre.

Relativement à ces conditions initiales, le pont peut s'effondrer mais peut tout aussi bien rester debout. La situation est aussi dépeinte dans le modèle arborescent par une structure de type $R$ qui représente le hasard objectif tout à fait comme en mécanique quantique. Dans le modèle, on trouve une distinction nette entre le déterminisme et le hasard, représentée par la différence entre les structures $U$ et $R$. 


\section{L'analogie avec Darwin}

A la fin de A Model of the Universe, j'ai fait une comparaison entre la méthode de raisonnement qu'on trouve dans le livre (qui se présente comme une version de l'argument appelé « inférence vers la meilleure explication ») et la méthode employée par Darwin dans L'Origine des espèces. Mais Marchildon ne voit pas de similitude entre les deux cas. Il note que la théorie de l'évolution n'est pas plus compliquée que sa rivale, la théorie de la création distincte des espèces, tandis que le modèle postule une structure de l'univers extrêmement complexe. Marchildon a raison - le modèle est très complexe. Mais la question que soulève la comparaison avec Darwin n'est pas de savoir si le modèle est plus ou moins complexe que son rival, mais celle de savoir si, comme dans le cas de la théorie de Darwin, on peut subsumer grâce à ce modèle un grand nombre d'explications relevant de différents domaines sous une seule et même hypothèse générale. Certes, l'hypothèse est complexe, mais, comme l'hypothèse de Darwin, elle me paraît foumir des explications de phénomènes nombreux et relevant de champs très divers.

Dans le domaine des propositions conditionnelles, le modèle fournit aussi une « sémantique probabiliste " qui jette de la lumière sur la différence, souvent remarquée mais toujours mal comprise, entre des propositions comme

(i) Si Oswald n'a pas tué Kennedy, quelqu'un d'autre l'a tué

et

(ii) Si Oswald n'avait pas tué Kennedy, quelqu'un d'autre l'aurait tué.

Comme le dit Marchildon, la structure du monde physique est ici invoquée pour tirer des conclusions dans le domaine de l'analyse du langage et dans celui de la sémantique. Je suis d'accord avec Marchildon pour dire que le réel est indépendant du langage, et je pense aussi que nous ne devons pas raisonner en inférant du dernier au premier. Mais justement, le modèle proposé ne se rend pas coupable d'utiliser un tel type d'argument fallacieux.

À propos des commentaires de Gauthier sur les inégalités de Bell et la non localité, je veux mettre au clair la différence dans le modèle arborescent entre les deux sortes d'évolution en mécanique quantique distinguées par von Neumann, c'est-à-dire l'évolution de Schrödinger et la mesure. Gauthier remarque à juste titre que la différence en est une entre faisceaux prismatiques ("prism stacks"). Quand il s'agit de l'évolution de la fonction d'onde, le système quantique fait face à un prisme ou arbre décénaire dans lequel le système se trouve dans le même état sur toutes les branches. Ceci est le modèle d'un changement d'état qui est $100 \%$ déterministe, car, peu importe quelle branche est sélectionnée pour devenir la branche actuelle, le système se retrouve dans le même état. Par contre, si le système fait face à un prisme de type $R$, dont plusieurs branches montrent le système dans un état $u$ et plusieurs autres dans un état différent $v$, les états $u$ et $v$ étant orthogonaux, alors dans ce 
cas le système subit un processus de mesure. Ce processus est le paradigme d'un processus indéterministe.

Dans l'expérience Bell-EPR, chacune des deux particules fait face à un processus de mesure, et les résultats ne sont pas indépendants l'un de l'autre mais révèlent une comélation très particulière. Quand il s'agit de deux photons dans un état quantique emmêlé, si l'on mesure les polarisations des deux photons par des appareils dont l'orientation est parallèle, alors, si le premier photon est mesuré « vertical », l'autre se montre toujours « horizontal ». Mais si le premier est « horizontal », l'autre est toujours « vertical ». Si les appareils ne sont pas disposés en parallèle mais sont séparés selon un angle $\emptyset$, et si le premier photon est mesuré " vertical ", alors la probabilité pour l'autre photon d'être mesuré $\emptyset+$ est $\sin ^{2} \emptyset$. Mais si le premier photon passe à la position " horizontale » plutôt que « verticale », alors la probabilité du deuxième photon d'être mesuré $\emptyset+$ est $\cos ^{2} \emptyset$ et non $\sin ^{2} \emptyset$. Peu importe que les deux événements où les photons passent par leurs appareils respectifs soient séparés de 10 mètres, de 10 kilomètres, voire de 10 millions de millions de kilomètres, la question se pose de savoir comment ces corrélations élégantes se produisent. Quelle explication peut-on en donner?

L'explication normale est que les résultats sont corrélés parce que les photons sont dans un mélange (superposition emmêlée) :

$$
\Phi=1 / \sqrt{ } 2(|v\rangle|h\rangle+|h>| v\rangle)
$$

Certes, l'hypothèse que les deux photons sont dans la superposition $\Phi$ explique les résultats observés en ce sens que les probabilités que l'on calcule à l'aide de $\Phi$, en employant les méthodes basées sur les vecteurs et les opérateurs dans l'espace de Hilbert, sont en parfait accord avec les probabilités observées. Mais le modele de l'espace de Hilbert est un modele théorique, et les moyens de calculer sont des moyens purement mathématiques. Il existe donc une explication mathématique ou théorique des corrélations observées, mais il n'existe pas d'explication physique. Nous n'avons rien identifié dans le monde physique, c'est-à-dire dans le monde de l'espace et du temps, qui pourrait expliquer l'interdépendance des résultats d'un coté et de l'autre : ni signal, ni transmission d'information d'une particule à l'autre qui semble correspondre au résultat de la mesure faite. Si une telle transmission avait lieu, l'autre processus de mesure, situé à une distance considérable, pourrait « connaître » les probabilités de ses propres résultats, afin de ne pas contredire les probabilités théoriques.

Pour souligner la nature précise de ce problème de corrélation, notons que chacune des deux observations à gauche et à droite est située hors du cône nul de l'autre, bien que leurs cônes nuls antérieurs se recoupent : 


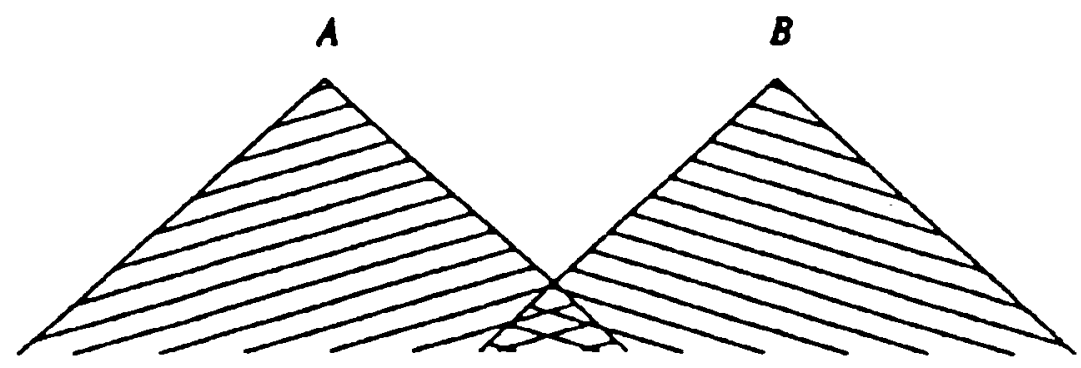

Selon la théorie restreinte de la relativité, aucun lien causal ne peut exister entre $A$ et $B$. La question se pose donc de savoir comment expliquer la cohérence des résultats. Dans la vie quotidienne, une corrélation observée entre deux événements simultanés est normalement expliquée par une cause commune. Si, par exemple, deux sifflets d'usine éclatent au même instant à Chicoutimi et à Montreal, cela s'explique par le fait que la synchronisation des horloges dans les deux villes constitue une cause commune. Cette synchronisation se trouve, bien entendu, dans l'intersection des deux cônes nuls des deux événements.

Mais dans le cas de l'expérience EPR, une explication de la corrélation par recours à un événement dans l'intersection des cônes nuls est exclue. La raison en est que les deux observations, c'est-à-dire les deux processus de mesure à $A$ et à $B$, sont des processus stochastiques ou indéterminés. Par conséquent, le résultat à $A$, c'est-à-dire le résultat " vertical » ou « horizontal », se produit sans aucun changement dans le cône nul de $A$. Le cône nul reste constant, et le résultat survient sans aucune dépendance par rapport aux événements du passé. Ceci est la nature d'un processus aléatoire. Également, à $B$, le résultat $\emptyset+$ ou $\emptyset$ - se produit sans aucune relation avec le contenu du cône nul de $B$. Mais, les deux résultats sont en corrélation l'un avec l'autre. Puisque la manifestation de chaque résultat est indépendante des événements dans son cône nul, il s'ensuit que la corrélation des deux résultats est également indépendante des événements dans l'intersection des deux cônes. On cherche donc en vain à trouver une explication des élégantes corrélations EPR dans le passé.

Mais si ce n'est dans le passé, où trouver alors une explication physique de ces corrélations non locales ? Revenons au modèle arborescent. Nous avons déjà remarqué que si le résultat du processus de mesure à gauche est « vertical », la probabilité du résultat « $\emptyset+»$ à droite est $\sin ^{2} \not \emptyset$, tandis que si le résultat à gauche est « horizontal », la probabilité de « $\emptyset+$ » à droite est $\cos ^{2} \phi$. Puisque les deux processus sont stochastiques, il faut trouver un modèle indéterministe pour expliquer l'influence du résultat à gauche sur le résultat à droite : 


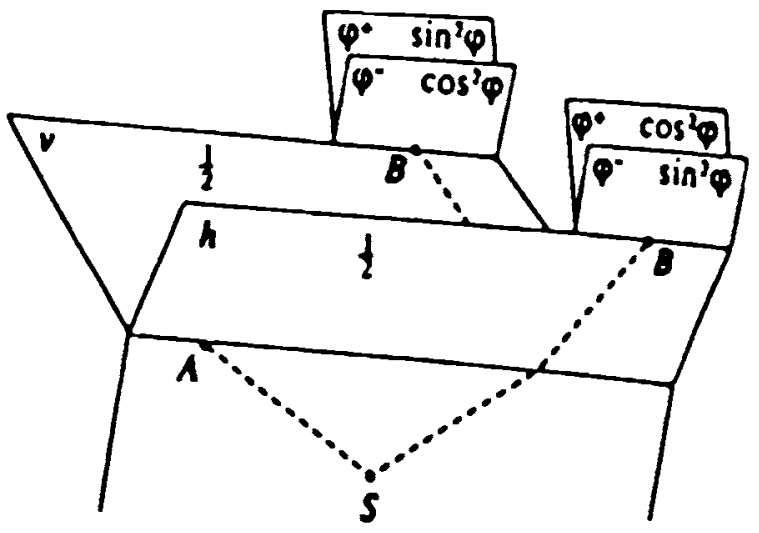

Dans ce modèle, le processus $R$ de mesure de von Neumann joue un rôle central. Si le résultat à gauche est « vertical », toutes les branches qui portent le résultat « horizontal » disparaissent, et sur la seule branche qui reste où apparaît le résultat « vertical », on voit que la probabilité du résultat « $\emptyset+$ » à droite est $\sin ^{2} \emptyset$. Mais si le résultat à gauche est « horizontal », alors les autres branches disparaissent et la probabilité de « $\emptyset+»$ est $\cos ^{2} \phi$ et non pas $\sin ^{2} \emptyset$. Cette disparition des branches dans le modele ( branch attrition ») est instantanée le long de la surface spatiale ondulée. Cela explique la manière dans laquelle un événement se produisant en un endroit peut influencer un événement se produisant en un autre endroit, sans violer le principe de la théorie de la relativité selon lequel aucune particule ni aucun signal ne peuvent se déplacer à une vitesse supérieure à celle de la lumière.

Pour terminer, j'aimerais dire que quelque sévères que soient les critiques présentées par Gauthier et Marchildon, elles sont bien pensées, bien raisonnées et bien formulées. Ces deux lecteurs ont compris l'esprit de mon ouvrage. J'espère néanmoins avoir répondu à leurs objections en ces quelques pages.

\section{B. Serge Robert}

J'en viens maintenant à la critique de Serge Robert, qui m'est venue plus tard que celles d'Yvon Gauthier et de Louis Marchildon, mais qui saisit très bien l'inspiration et l'esprit philosophique de mon ouvrage. Les commentaires de Serge Robert qui touchent le coeur de mon point de vue philosophique sont les suivants.

(1) Entre le futur et le passé, c'est-à-dire entre les branches du modèle arborescent et le tronc, il y a une différence profonde mais évanescente. Parmi toutes les branches qui sortent du premier noeud de l'arbre quadridimensionnel, c'est-à-dire le présent, une et seulement une branche sera choisie pour 
devenir le prolongement du passé. Par conséquent, une «petite partie » du futur devient le passé, et le passé est entièrement composé de "morceaux » de l'avenir. (J'espère que les mots " partie " et " morceaux " ne feront pas naître plus de problèmes qu'ils n'en résolvent.) Robert soulève la question de savoir si la distinction faite par le modèle entre futur et passé n'est pas trop " tranchée » et absolue, et il avance l'idée que le passé et le futur sont probablement beaucoup plus entremêlés que le modèle ne le prétend.

Robert a raison. Le passé et le futur sont liés ensemble d'une façon incontestable, et la distinction tronc/branches du modèle risque d'affaiblir le rapport entre les deux. En même temps, Robert reconnaît que le dynamisme du modele crée des liens entre le passé et le futur en disant que « la chute du futur dans le passé est assurée par le simple passage du temps ». En effet, il aurait pu s'exprimer de façon plus forte encore puisque, dans le modèle, la chute du futur n'est pas simplement «assurée » par lui : elle constitue le passage du temps. Ce dermier n'est rien d'autre que le choix ou la sélection d'une branche parmi la multitude qui constitue l'avenir, et la disparition du reste. C'est le fameux «émondage " des branches qui fait mal aux philosophes, eux qui préfèrent regarder l'univers sub specie aternitatis; mais c'est un élément objectif et inéliminable du modèle.

Si je peux me permettre une prédiction, je dirai que, pendant les prochains deux cents ans, il y aura deux sujets de débat vigoureux entre philosophes : (i) la question de savoir s'il existe une différence absolue entre la connaissance humaine et la " connaissance " artificielle d'un ordinateur ou d'un robot, et (ii) la question de savoir s'il est possible de donner une description précise et scientifique de l'écoulement du temps. L'objet de ce dernier débat concernera la possibilité d'identifier une caractéristique réelle du monde qui corresponde à la notion de "passage temporel », sans quoi cette idée restera dans le domaine de la métaphore et de la poésie.

Continuant sur la même voie, Robert remarque que le fait que les objets perdurent et ne soient pas anéantis par le passage du temps, c'est-à-dire par l'émondage des branches, constitue un deuxième lien entre le passé et le futur. La chaise sur laquelle je m'assieds aujourd' hui est la même chaise qui m'a soutenu hier et qui me soutiendra probablement demain. Elle fait partie de toutes les branches qui constituent l'avenir (sauf peut-être celles correspondant à un tremblement de terre entraînant la destruction de ma maison), et l'identité de ces « chaises futures ", chacune dans sa branche, est constituée par une seule origine commune dans le tronc du modèle, où la chaise a été construite par un menuisier. Dans sa description quadridimensionelle, "la " chaise est un objet à la fois multiple, continu et folié, enfoncé dans le tronc et dans chaque branche du modèle. Chaque objet, par conséquent, réunit, dans sa forme arborescente et dynamique, le passé avec l'avenir.

(2) Robert note que la structure arborescente fournit l'ontologie d'un modèle sémantique pour deux sortes d'énoncés conditionnels : ceux du type A, comme «Si Hitler avait envahi l'Angleterre en 1940, il aurait gagné la 
guerre », et ceux du type B, comme «Si Shakespeare n'a pas écrit Hamlet, quelqu'un d'autre l'a fait ". Ces deux conditionnels contrefactuels ont des propriétés sémantiques bien différentes. Le premier possède une valeur de probabilité mais n'a pas de valeur de vérité ; le deuxième a une valeur de vérité mais n'a pas de valeur de probabilité. D'où vient cette différence, remarquée par plusieurs philosophes mais expliquée jusqu'à maintenant par aucun d'entre eux? Robert observe que la valeur de probabilité des conditionnels de type A est fondée sur la proportionnalité des ensembles de branches dans le modèle, tandis que la valeur de vérité de ceux de type B est déterminée par la perte des branches. Ceci constitue une différence fondamentale : la sémantique des conditionnels de type A n'est pas du tout semblable à la sémantique des conditionnels de type $B$.

Robert reconnaît la nécessité de distinguer les deux bases sémantiques, mais il regrette que la distinction soit tellement brusque que nous risquions de perdre le rapport traditionnel entre probabilité et vérité. Du point de vue classique, «vrai » et «faux " sont les limites d'une gamme de valeurs intermédiaires de probabilité, et « vrai » équivaut à " probable à 100\%». Mais dans le modèle, la vérité de «Si Shakespeare n'a pas écrit Hamlet, quelqu'un d'autre l'a fait » n'est pas basée sur le fait que chaque noeud où Shakespeare n'écrit pas Hamlet est suivi par une proportion de $100 \%$ de branches sur lesquelles quelqu'un d'autre écrit Hamlet. Non. La vérité du conditionnel est basée sur un événement contingent, produit par l'émondage des branches, c'est-à-dire l'événement correspondant au fait que quelqu'un a écrit Hamlet. Ceci est un fait historique, et n'a pas été produit par l'opération d'une loi de la nature. La probabilité initiale de cette circonstance, qu'un écrivain quelconque écrirait Hamlet, était sans doute très petite. Mais, une fois survenu, l'événement ne permet à la proposition du type B ci-haut d'avoir qu'une seule valeur, c'est-àdire la valeur «vraie». Pour cette raison, les valeurs de vérité de ces conditionnels n'ont rien à voir avec la probabilité, ni avec la proportion des ensembles de branches dans le modèle. Par contre, la sémantique des énoncés de type A est entièrement basée sur la proportionnalité des branches.

(3) Passons maintenant aux lois de la nature. Robert note qu' une loi est considérée comme un «pattern » de branchement. Ainsi, dit-il, on assiste à une loi absolue si, à partir d'un noeud, toutes les branches aboutissent au même résultat, ou, ce qui est plus souvent le cas, à une loi probabiliste si certaines branches arrivent à un résultat et d'autres pas. Ceci est vrai, mais il faut ajouter un élément essentiel, c'est-à-dire que ce «pattern » doit être reproduit dans chaque partie du modele s'il s'agit d'une vraie loi, soit absolue, soit probabiliste. Ainsi, pour qu'un événement de type $X$ soit suivi par un événement de type $Y$ avec une probabilité de $70 \%$, il faut que chaque noeud dans le modèle entier qui contient $\mathrm{X}$ soit suivi par une proportion égale de branches qui contiennent $Y$. Il est impossible, objectera-t-on, de vérifier en pratique une condition aussi rigoureuse. Cela est vrai mais n'importe pas, car l'existence des lois de la nature ne dépend pas de notre capacité de les connaître. Robert dit très 
justement que cette interprétation des lois fournie par le modele est objectiviste, et non subjectiviste. Elle fonde les lois ontologiquement plutôt qu'épistémologiquement. Une conséquence de cette approche est la suivante : les lois de la nature évoluent avec le passage du temps. Plus il y a perte de branches, plus le nombre de lois augmente. (Robert écrit « diminue », mais ceci est incorrect : un arbre plus petit satisfait à ces conditions plus facilement qu' un grand arbre.) En somme, le modelle donne lieu à une conception des lois de la nature qui est à la fois objectiviste et dynamique.

(4) Dans sa section 5, Robert distingue le «branch pattern» qui, dans le modèle, constitue une loi, de la "branched shape " manifestée par une espèce d'objet ou d'organisme. Prenons un moment pour clarifier cette distinction.

Un « branch pattern » est une proportion constante, partout dans le modèle, de branches de type $\mathrm{Y}$ au-dessus de noeuds de type $\mathrm{X}$. Le «branched shape " d'un objet ou d'un organisme est la forme quadridimensionnelle de cet objet ou de cet organisme, reproduite grosso modo sur chaque branche dans laquelle l'objet existe. (Rappelons que tous les volumes quadridimensionnels d'un seul objet, dans toutes les branches, se réunissent à l'origine de l'objet.) Si nous prenons, par exemple, le cas d'un papillon, nous trouverons qu'une «branched shape » très particulière caractérise cet insecte. Au début, il possède la forme quadridimensionnelle d'un oeuf (avec extension temporelle, bien entendu); plus tard, c'est-à-dire à un moment plus éloigné de l'origine, il a la forme d'une chenille (qu'on peut faire émerger en faisant dans le volume quadrimensionnel une section orthogonale à l'axe du temps); éventuellement, le volume quadridimensionnel manifeste la forme dynamique et énergique d'un papillon qui vole de fleur en fleur. Peut-être dans quelques branches le papillon perd-il une patte ou endommage-t-il une de ses ailes. Peu importe. Il reste toujours un papillon, gardant cette propriété essentielle sur toutes les branches où il existe. En effet, chaque espèce ou " natural kind" manifeste sa propre forme quadridimensionnelle, et l'ensemble de ces formes constitue l'ontologie des individus du modèle arborescent.

(5) Pour conclure, je dirai quelques mots de la question de la décision, de l'intentionnalité et du libre arbitre. Robert reconnaût que, si notre point de départ est une métaphysique indéterministe, comme celle du modèle arborescent, le problème du libre arbitre ne consiste pas à le rendre compatible avec le déterminisme (le problème classique du libre arbitre) mais avec l'indéterminisme et le hasard. L'argument se poursuit comme suit.

Dans la tradition philosophique, la liberté d'action est aussi difficile à réconcilier avec le hasard qu'avec le déterminisme. Un être dont le comportement serait réglé par un processus comme tirer à pile ou face n'est pas plus libre dans le sens humain du terme qu'un robot ou un mécanisme dont les actions sont programmées et prévisibles à cent pour cent. Existe-t-il une via media entre le déterminisme strict et le hasard pur et simple ? Oui, pour un organisme qui cherche à faire analyser ses actions avec l'aide du modèle arborescent. 
Supposons, par exemple, que cet organisme se trouve à un noeud dans le modèle qui lui donne le choix entre trois actions différentes, $\mathrm{A}, \mathrm{B}$ et $\mathrm{C}$. L'organisme délibère, et, après une période de délibération, il tranche et choisit l'action $B$. Puisque chacune des options $A, B$ et $C$ reste possible jusqu'au moment de la décision, le choix d'aucune de ces trois suites ne peut être expliqué de façon déterministe. Mais, en même temps, la décision faite par l'organisme en faveur de B n'est pas aléatoire non plus. Le choix a été fait pour une raison. Cette raison n'est pas une cause physique, parce qu'autrement la décision serait déterminée d'avance, ce qui n'est pas le cas. La raison est une raison intentionnelle, et c'est justement la possibilité de fournir une explication intentionnelle de la décision qui la rend non aléatoire. Une décision faite par un organisme dans le contexte du modèle arborescent est donc un événement qui ne résulte ni du déterminisme, ni du hasard mais qui se prête à une explication intentionnelle qui n'a rien à voir avec la causalité physique. Ceci est la via media ouverte par le modèle entre les deux extrêmes du déterminisme et de l'indéterminisme simple. Cette via media rend possible une liberté d'action basée sur la notion aristotélicienne de

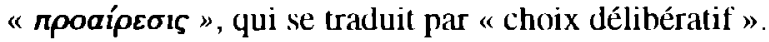

Tout cela est reconnu par Robert, qui termine sa critique en se demandant si l'analyse de la liberté que je viens de faire ne va pas trop loin dans le sens de l'indéterminisme et ne néglige pas les facteurs qui déterminent nos actions et « orientent l'existence humaine au profit d'une survalorisation des capacités de la volonté ". En effet, je ne voudrais pencher trop fortement ni de l'un ni de l'autre côté, mais plutôt garder un équilibre entre déterminisme et volontarisme. Peut-être la notion de « rpoaípєoı » viendra-t-elle à notre aide ici. Quand nous prenons une décision, cette décision n'est pas basée sur un choix totalement arbitraire. Notre décision d'aujourd'hui est le produit de mille ou même d'un million de décisions passées; nous sommes en un certain sens construits par nos choix antérieurs. Si vous me connaissez bien, vous pourrez sans doute prédire la majorité de mes décisions. Mais ceci n'équivaut pas à du déterminisme. Mon pouvoir de décision reste à moi, et je peux toujours vous surprendre en choisissant une option qui, en vertu de votre estimation, est très peu probable. Donc, la notion du choix intentionnel, qui est centrale pour l'analyse de la liberté humaine dans $A$ Model of the Universe, permet de conserver un équilibre essentiel entre les sphères de la détermination et de la créativité.

Départenent de philosophie

Université McGill 